\title{
Т.П. Гой
}

\section{СПЕЦИАЛЬНЫЕ ФУНКЦИИ, ПОСТРОЕННЫЕ С ПОМОЩЬЮ ВОЗРАСТАЮЩИХ И ЦЕНТРАЛЬНЫХ ФАКТОРИАЛЬНЫХ СТЕПЕНЕЙ}

\begin{abstract}
Изучаются неэлементарные функции типа интегралов Френеля, определенные в виде степенных рядов с использованием возрастающих и центральных факториальных степеней. Установлены некоторые свойства этих функций, приведены их графики. Выведены обыкновенные дифференциальные уравнения, решениями которых являются новые функции.
\end{abstract}

Ключевые слова: возрастающая факториальная степень, иентральная факториальная степень, интеграль Френеля, обобщенная гипергеометрическая функиия, задача Коши.

Математические модели многих процессов и явлений часто приводят к задачам, точные решения которых классическими методами получить невозможно. Увеличение количества неэлементарных функций приводит к расширению круга задач, которые могут быть решены в замкнутом виде. При этом особое внимание уделяется исследованию новых функций с целью их использования для решения сложных теоретических и практических задач.

Тригонометрические функции $\cos x, \sin x$ задаются как степенные ряды

$$
\cos x=\sum_{n=0}^{\infty} \frac{(-1)^{n}}{(2 n) !} x^{2 n}, \quad \sin x=\sum_{n=0}^{\infty} \frac{(-1)^{n}}{(2 n+1) !} x^{2 n+1},
$$

определенные с помощью факториалов (убывающих факториальных степеней). Заменив в этих рядах убывающие факториальные степени соответствующими возрастающими факториальными степенями, авторы [1] изучили новые неэлементарные функции действительной переменной $\operatorname{Cos} x, \operatorname{Sin} x$. Аналогично, в $[2,3]$ представлено исследование функций действительной переменной $\operatorname{Cosc} x, \operatorname{Sinc} x$, построенных с помощью центральных факториальных степеней. Показано, в частности, что новые функции являются решениями обыкновенных линейных дифференциальных уравнений второго (функции $\operatorname{Cos} x, \operatorname{Sin} x$ ) и третьего (функции $\operatorname{Cosc} x, \operatorname{Sinc} x)$ порядка с полиномиальными коэффициентами.

В [4] рассмотрены неэлементарные функции типа интегралов Френеля, определенные в виде степенных рядов с использованием возрастающих факториальных степеней. Автором [5-8] изучаются некоторые другие подобного рода функции действительного и комплексного переменного.

\section{1. Факториальные степени}

Для произвольных чисел $x \in \mathbf{R}$ и $m \in \mathbf{N}$ факториальной степенью $m$ с шагом $k \in \mathbf{R}$ называют выражение [9]

$$
x^{m\{k\}}=x(x+k)(x+2 k) \cdot \ldots \cdot(x+(m-1) k) .
$$


Факториальную степень называют возрастающей, если $k>0$, и убывающей, если $k<0$. В случае $k=0$ имеем обыкновенную степень, т.е. $x^{m\{0\}}=x^{m}$. По умолчанию, $x^{0\{k\}}=1$. Возрастающую факториальную степень $m$ с шагом 1 и убывающую факториальную степень $m$ с шагом $(-1)$ будем обозначать через $x^{\bar{m}}$ и $x^{\underline{m}}$ соответственно, т.е.

$$
\begin{aligned}
& x^{\bar{m}} \equiv x^{m\{1\}}=x(x+1) \cdot \ldots \cdot(x+m-1), \\
& x^{\underline{m}} \equiv x^{m\{-1\}}=x(x-1) \cdot \ldots \cdot(x-m+1) .
\end{aligned}
$$

Очевидно, что $n !=1^{\bar{n}}=n^{\underline{n}}$.

Основные свойства возрастающих и убывающих факториальных степеней выражаются с помощью формул

$$
\bar{\Delta} x^{\bar{m}}=m x^{\overline{m-1}}, \quad \Delta x^{\underline{m}}=m x^{\underline{m-1}},
$$

где $\Delta f(x)=f(x+1)-f(x)$ - разность функции $f(x)$, а $\bar{\Delta} f(x)=f(x)-f(x-1)$ опаздывающая разность этой функции.

Комбинаторный закон двойственности возрастающих и убывающих факториальных степеней выражается с помощью равенств

$$
(-m)^{n\{-k\}}=(-1)^{n} m^{n\{k\}}, \quad(-m)^{n\{k\}}=(-1)^{n} m^{n\{-k\}} .
$$

Для произвольных чисел $x \in \mathbf{R}$ и $m \in \mathbf{N}$ иентральной факториальной степенью $m$ с шагом $k>0$ называют выражение [10]

$$
x^{m[k]}=x\left(x+\frac{m k}{2}-k\right)\left(x+\frac{m k}{2}-2 k\right) \cdot \ldots \cdot\left(x-\frac{m k}{2}+k\right),
$$

причем $x^{0[k]}=1$. Центральную факториальную степень $m$ с шагом 1 будем обозначать через $x^{[m]}$, например

$$
\begin{gathered}
x^{5[1]} \equiv x^{[5]}=\left(x-\frac{3}{2}\right)\left(x-\frac{1}{2}\right) x\left(x+\frac{1}{2}\right)\left(x+\frac{3}{2}\right), \\
x^{6[1]} \equiv x^{[6]}=(x-2)(x-1) x^{2}(x+1)(x+2) .
\end{gathered}
$$

Для центральных факториальных степеней с шагом 1 имеет место формула

$$
\delta x^{[m]}=m x^{[m-1]},
$$

аналогичная соответственным формулам для возрастающих и убывающих факториальных степеней. Здесь $\delta f(x)=f(x+1 / 2)-f(x-1 / 2)$ - центральная разность функции $f(x)$.

Легко показать, что $x^{[m]}=x(x+m / 2-1)^{\underline{m}}$. Другие свойства и некоторые применение факториальных степеней можно найти, например, в [11] - [14].

Отметим, что возрастающим, убывающим и центральным факториальным степеням в комбинаторном анализе часто присуща двойственность: если комбинаторная задача приводит к тождеству с использованием, например, убывающих факториальных степеней, то обычно существует содержательная комбинаторная задача, приводящая к двойственному тождеству с участием возрастающих или центральных факториальных степеней. 


\section{2. Неэлементарные функции $\operatorname{Cos} x, \operatorname{Sin} x, \operatorname{Cosc} x, \operatorname{Sinc} x$, определенные с помощью возрастающих и центральных факториальных степеней}

Обозначим через $\operatorname{Cos} x, \operatorname{Sin} x, \operatorname{Cosc} x, \operatorname{Sinc} x$ функции действительного переменного, определенные с помощью степенных рядов [1,2]:

$$
\begin{gathered}
\operatorname{Cos} x=\sum_{n=0}^{\infty} \frac{(-1)^{n}}{(2 n)^{2 n}} x^{2 n}=1-\frac{x^{2}}{2 \cdot 3}+\frac{x^{4}}{4 \cdot 5 \cdot 6 \cdot 7}-\frac{x^{6}}{6 \cdot 7 \cdot 8 \cdot 9 \cdot 10}+\ldots ; \\
\operatorname{Sin} x=\sum_{n=0}^{\infty} \frac{(-1)^{n}}{(2 n+1)^{\overline{2 n+1}}} x^{2 n+1}=\frac{x}{1}-\frac{x^{3}}{3 \cdot 4 \cdot 5}+\frac{x^{5}}{5 \cdot 6 \cdot 7 \cdot 8 \cdot 9}-\ldots ; \\
\operatorname{Cosc} x=\sum_{n=0}^{\infty} \frac{(-1)^{n}}{(2 n)^{[2 n]}} x^{2 n}=1-\frac{x^{2}}{2 \cdot 2}+\frac{x^{4}}{3 \cdot 4 \cdot 4 \cdot 5}-\frac{x^{6}}{4 \cdot 5 \cdot 6 \cdot 6 \cdot 7 \cdot 8}+\ldots ; \\
\operatorname{Sinc} x=\sum_{n=0}^{\infty} \frac{(-1)^{n}}{(2 n+1)^{[2 n+1]}} x^{2 n+1}=\frac{x}{1}-\frac{x^{3}}{\frac{5}{2} \cdot 3 \cdot \frac{7}{2}}+\frac{x^{5}}{\frac{7}{2} \cdot \frac{9}{2} \cdot 5 \cdot \frac{11}{2} \cdot \frac{13}{2}}-\ldots
\end{gathered}
$$

Легко убедиться, что

$$
\begin{aligned}
& \operatorname{Cos} x=1+\sum_{n=1}^{\infty} \frac{(-1)^{n}(2 n-1) !}{(4 n-1) !} x^{2 n}, \quad \operatorname{Sin} x=\sum_{n=1}^{\infty} \frac{(-1)^{n-1}(2 n-2) !}{(4 n-3) !} x^{2 n-1} ; \\
& \operatorname{Cosc} x=1+\frac{1}{2} \sum_{n=1}^{\infty} \frac{(-1)^{n}(n-1) !}{(3 n-1) !} x^{2 n}, \quad \operatorname{Sinc} x=\sum_{n=1}^{\infty} \frac{(-1)^{n} 4^{n}(2 n-1) ! !}{(6 n+1) ! !} x^{2 n+1} .
\end{aligned}
$$

В [1 - 3] доказано, что

$$
\begin{gathered}
\operatorname{Cos} x=1+2 \sqrt{x}\left(\cos \frac{x}{4} S\left(\frac{\sqrt{x}}{2}\right)-\sin \frac{x}{4} C\left(\frac{\sqrt{x}}{2}\right)\right) \\
\operatorname{Sin} x=2 \sqrt{x}\left(\cos \frac{x}{4} C\left(\frac{\sqrt{x}}{2}\right)+\sin \frac{x}{4} S\left(\frac{\sqrt{x}}{2}\right)\right),
\end{gathered}
$$

где $C(p)=\int_{0}^{p} \cos t^{2} d t, S(p)=\int_{0}^{p} \sin t^{2} d t-$ интегралы Френеля [15], а также установлена связь между функциями $\operatorname{Cos} x, \operatorname{Sin} x, \operatorname{Cosc} x, \operatorname{Sinc} x$ и обобщенной гипергеометрической функцией ${ }_{1} F_{2}\left(a_{1} ; b_{1}, b_{2} ; z\right)$ с помощью формул

$$
\begin{array}{ll}
\operatorname{Cos} x=1-\frac{x^{2}}{6} \cdot{ }_{1} F_{2}\left(1 ; \frac{5}{4}, \frac{7}{4} ;-\frac{x^{2}}{64}\right), & \operatorname{Sin} x=x \cdot{ }_{1} F_{2}\left(1 ; \frac{3}{4}, \frac{5}{4} ;-\frac{x^{2}}{64}\right), \\
\operatorname{Cosc} x=1-\frac{x^{2}}{4} \cdot{ }_{1} F_{2}\left(1 ; \frac{4}{3}, \frac{5}{3} ;-\frac{x^{2}}{27}\right), & \operatorname{Sinc} x=x \cdot{ }_{1} F_{2}\left(1 ; \frac{5}{6}, \frac{7}{6} ;-\frac{x^{2}}{27}\right) .
\end{array}
$$

Напомним, что обобщенная гипергеометрическая функция

$$
{ }_{s} F_{q}\left(a_{1}, \ldots, a_{s} ; b_{1}, \ldots, b_{q} ; z\right)
$$


определятся как сумма обобщенного гипергеометрического ряда [16]

$$
{ }_{s} F_{q}\left(a_{1}, \ldots, a_{s} ; b_{1}, \ldots, b_{q} ; z\right)=\sum_{n=0}^{\infty} \frac{a_{1}^{\bar{n}} \cdot \ldots \cdot a_{s}^{\bar{n}}}{b_{1}^{\bar{n}} \cdot \ldots \cdot b_{q}^{\bar{n}}} \cdot \frac{z^{n}}{n !}
$$

в области его сходимости, где $a_{1}^{\bar{n}}, \ldots, a_{s}^{\bar{n}}, b_{1}^{\bar{n}}, \ldots, b_{q}^{\bar{n}}$ - возрастающие факториальные степени с шагом 1.

На рис. $1-4$ приведены графики функций $\operatorname{Cos} x, \operatorname{Sin} x, \operatorname{Cosc} x, \operatorname{Sinc} x$. На рис. 1 , 2 пунктиром проведены параболы $(y+1)^{2}= \pm \pi x$ и $y^{2}= \pm \pi x$ соответственно, а на рис. 3,4 - соответственно параболы $\left(y+\frac{1}{2}\right)^{2}= \pm \frac{7 \pi x}{12}$ и $y^{2}= \pm \frac{7 \pi x}{12}$.

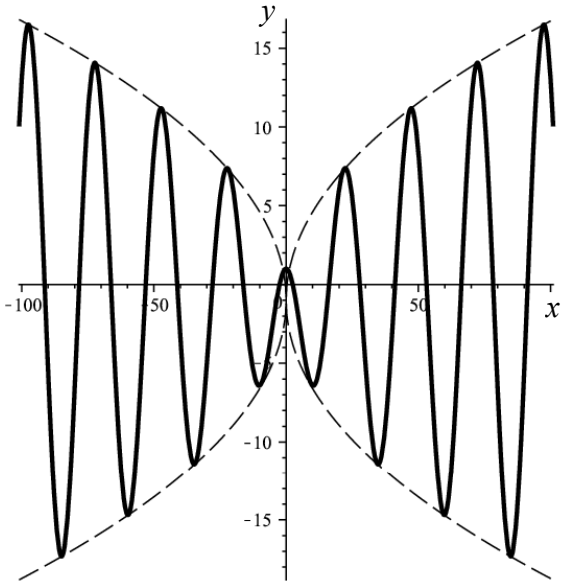

Рис. 1. График функции $y=\operatorname{Cos} x$

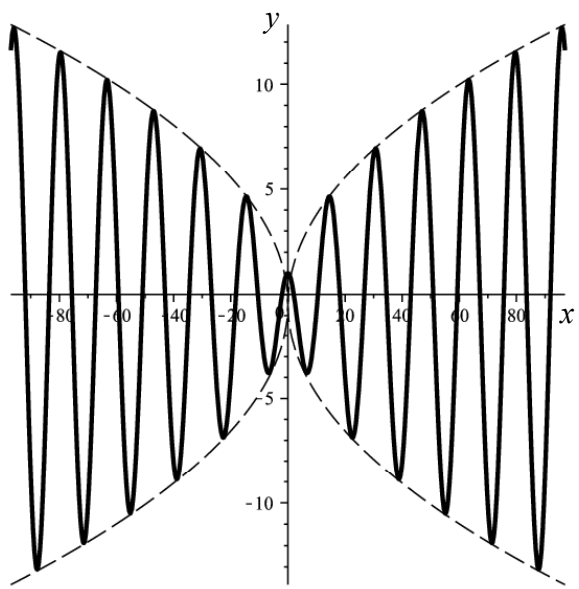

Рис. 3. График функции $y=\operatorname{Cosc} x$

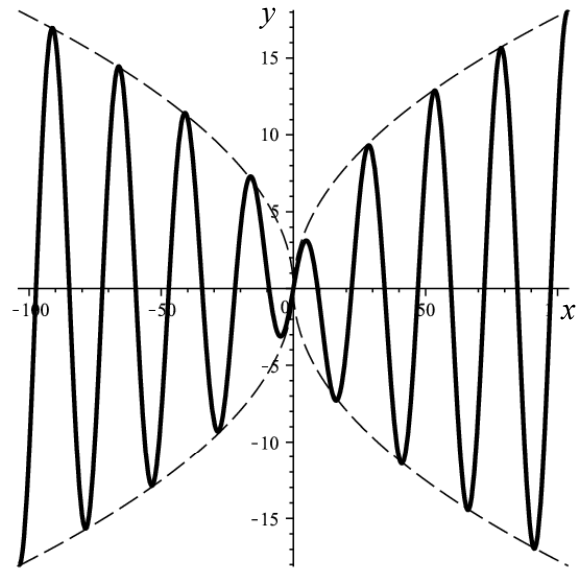

Рис. 2. График функции $y=\operatorname{Sin} x$

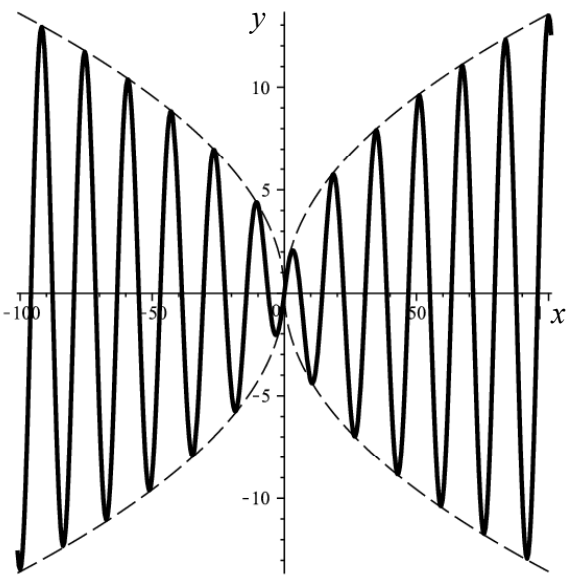

Рис. 4. График функции $y=\operatorname{Sinc} x$ 
3. Неэлементарные функции типа интегралов Френеля, определенные с помощью возрастающих факториальных степеней

Обозначим через $S_{1}(x), C_{1}(x)$ функции

$$
\begin{aligned}
& C_{1}(x)=\int_{0}^{x} \operatorname{Cos} t^{2} d t ; \\
& S_{1}(x)=\int_{0}^{x} \operatorname{Sin} t^{2} d t .
\end{aligned}
$$

Из формул (9), (10), учитывая (5), получаем разложения функций $S_{1}(x), C_{1}(x)$ в степенные ряды, абсолютно сходящиеся на всей числовой оси:

$$
\begin{gathered}
C_{1}(x)=x+\sum_{n=1}^{\infty} \frac{(-1)^{n}(2 n-1) !}{(4 n-1) !(4 n+1)} x^{4 n+1} ; \\
S_{1}(x)=\sum_{n=1}^{\infty} \frac{(-1)^{n-1}(2 n-2) !}{(4 n-3) !(4 n-1)} x^{4 n-1} .
\end{gathered}
$$

Графики функций $C_{1}(x), S_{1}(x)$ приведены на рис. 5,6 .

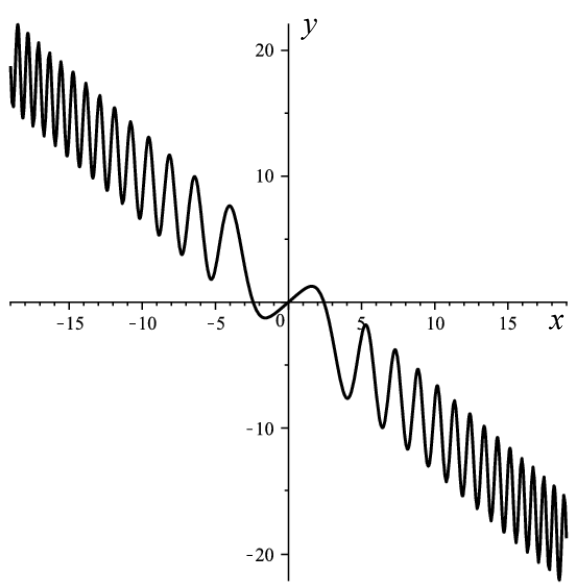

Рис. 5. График функции $y=C_{1}(x)$

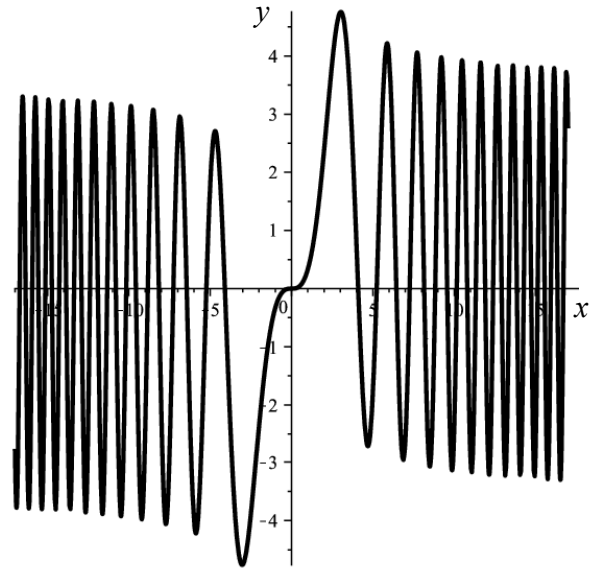

Рис. 6. График функции $y=S_{1}(x)$

Теорема 1. [4] Для всех $x \in \mathbf{R}$ имеют место тождества

$$
\begin{gathered}
C_{1}(x)=4\left(\cos \frac{x^{2}}{4} C\left(\frac{x}{2}\right)+\sin \frac{x^{2}}{4} S\left(\frac{x}{2}\right)\right)-x, \\
S_{1}(x)=4\left(\sin \frac{x^{2}}{4} C\left(\frac{x}{2}\right)-\cos \frac{x^{2}}{4} S\left(\frac{x}{2}\right)\right),
\end{gathered}
$$

где $C(p), S(p)-$ интеграль Френеля.

На рис. 7-12 представлены графики функций комплексного переменного $C_{1}(z), S_{1}(z)$, где $z=x+i y$. 


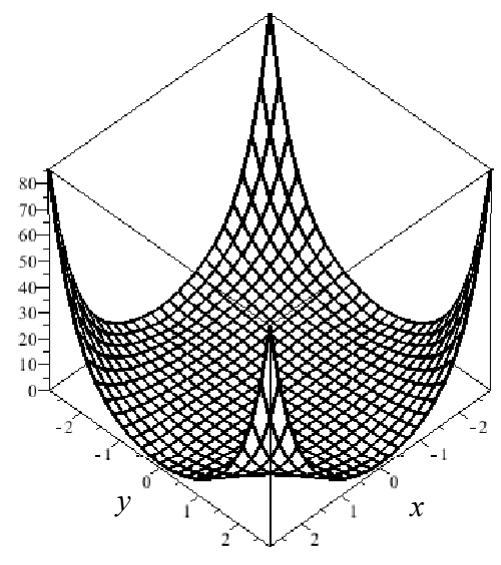

Рис. 7. График функции $\left|C_{1}(z)\right|$

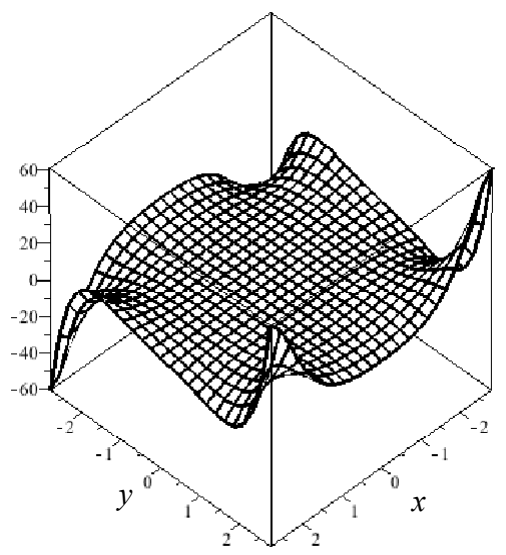

Рис. 9. График функции $\operatorname{Im}\left(C_{1}(z)\right)$

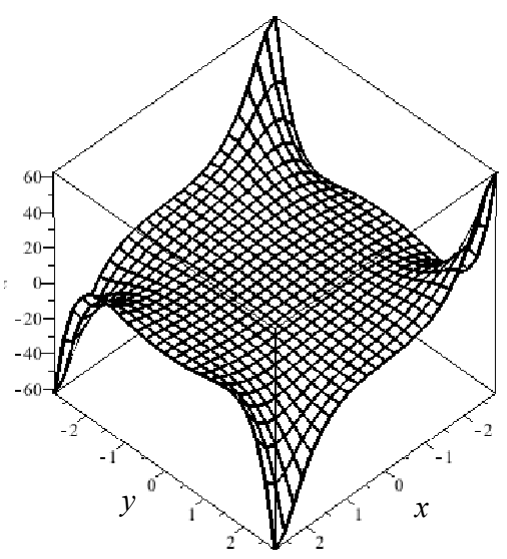

Рис. 11. График функции $\operatorname{Re}\left(S_{1}(z)\right)$

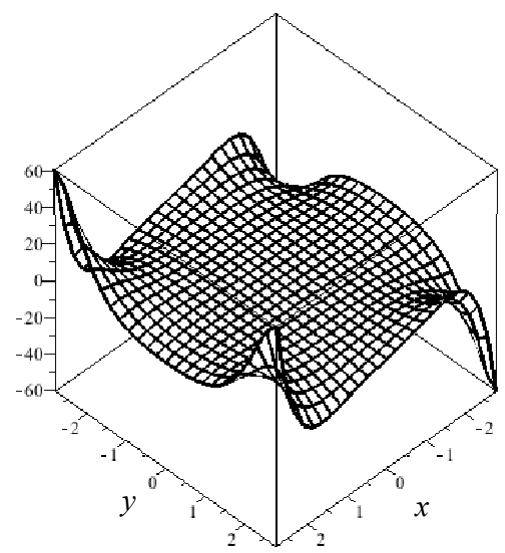

Рис. 8. График функции $\operatorname{Re}\left(C_{1}(z)\right)$

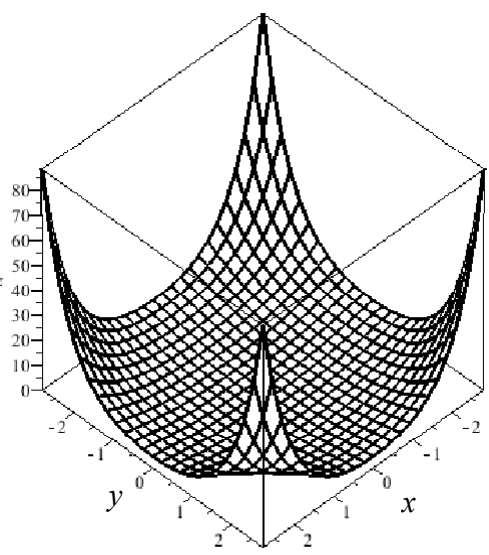

Рис. 10. График функции $\left|S_{1}(z)\right|$

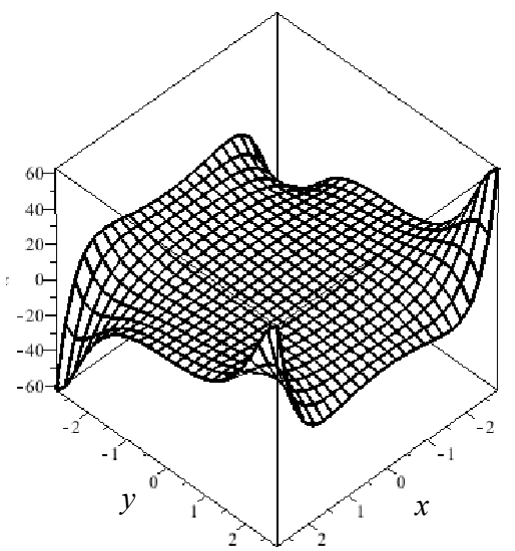

Рис. 12. График функции $\operatorname{Im}\left(S_{1}(z)\right)$ 
Покажем, что функции $C_{1}(x)$ и $S_{1}(x)$ являются решениями линейных неоднородных дифференциальных уравнений второго порядка с переменными коэффициентами.

Теорема 2. Функциии $C_{1}(x)$ и $S_{1}(x)$ - решения соответственно таких задач Коuи:

$$
\begin{gathered}
4 x y^{\prime \prime}-4 y^{\prime}+x^{3} y=-x^{4}-4, \quad y(0)=0, \quad y^{\prime}(0)=1 ; \\
4 x y^{\prime \prime}-4 y^{\prime}+x^{3} y=4 x^{2}, \quad y(0)=0, \quad y^{\prime}(0)=0 .
\end{gathered}
$$

Доказательство. То, что функции $C_{1}(x)$ и $S_{1}(x)$ удовлетворяют начальные условия из (13), (14), следует соответственно из формул (11) и (12). Докажем, что эти функции являются решениями соответствующих дифференциальных уравнений.

Обозначим

$$
\begin{aligned}
& \alpha(x) \equiv \sin \left(x^{2} / 4\right) S(x / 2)+\cos \left(x^{2} / 4\right) C(x / 2), \\
& \beta(x) \equiv \sin \left(x^{2} / 4\right) C(x / 2)-\cos \left(x^{2} / 4\right) S(x / 2) .
\end{aligned}
$$

Тогда из формулы (9), учитывая (7), находим производные функции $C_{1}(x)$ :

$$
C_{1}^{\prime}(x)=1-2 x \beta(x), \quad C_{1}^{\prime \prime}(x)=-2 \beta(x)-x^{2} \alpha(x) .
$$

Теперь дифференциальное уравнение из (13) легко получаем, исключая из (7), (15) выражения $\alpha(x)$ и $\beta(x)$.

То, что функция $S_{1}(x)$ является частным решением дифференциального уравнения из (14), доказывается аналогично, путем исключения выражений $\alpha(x), \beta(x)$ из (8) и следующих формул для производных функции $S_{1}(x)$ :

$$
S_{1}^{\prime}(x)=2 x \alpha(x), \quad S_{1}^{\prime \prime}(x)=x-x^{2} \beta(x)+2 \alpha(x) .
$$

\section{4. Неэлементарные функции типа интегралов Френеля, определенные с помощью центральных факториальных степеней}

Обозначим через $C_{2}(x)$ и $S_{2}(x)$ функции

$$
\begin{aligned}
C_{2}(x) & =\int_{0}^{x} \operatorname{Cosc} t^{2} d t ; \\
S_{2}(x) & =\int_{0}^{x} \operatorname{Sinc} t^{2} d t .
\end{aligned}
$$

Из (16), (17), учитывая формулы (6), получаем следующие разложения функций $C_{2}(x), S_{2}(x)$ в степенные ряды:

$$
\begin{gathered}
C_{2}(x)=x+\frac{1}{2} \sum_{n=1}^{\infty} \frac{(-1)^{n}(n-1) !}{(3 n-1) !(4 n+1)} x^{4 n+1} ; \\
S_{2}(x)=\sum_{n=1}^{\infty} \frac{(-1)^{n} 16^{n}(2 n) !(3 n) !}{n !(6 n+1) !(4 n+3)} x^{4 n+3},
\end{gathered}
$$

абсолютно сходящиеся при каждом действительном $x$. 
Графики функций $C_{2}(x)$ и $S_{2}(x)$ приведены на рис. 13 и 14.

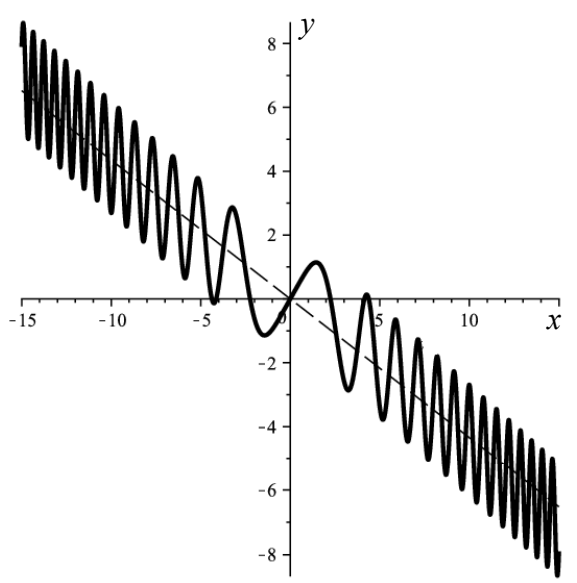

Рис. 13. График функции $y=C_{2}(x)$

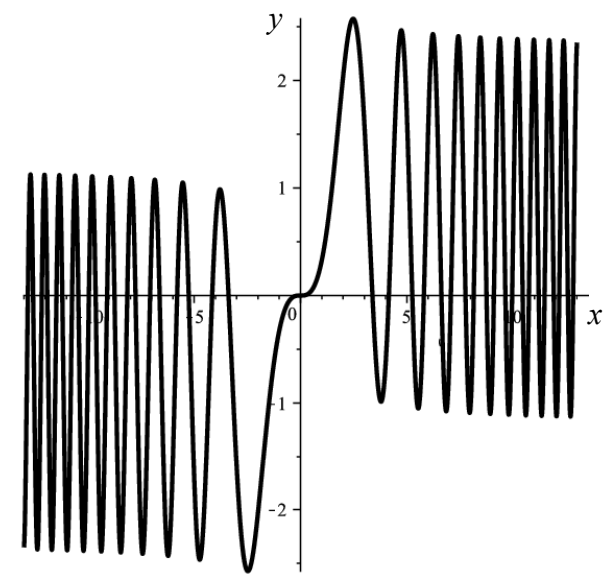

Рис. 14. График функции $y=S_{2}(x)$

Следующая теорема устанавливает связь между интегральными функциями $C_{2}(x), S_{2}(x)$ и обобщенной гипергеометрической функцией ${ }_{2} F_{3}\left(a_{1}, a_{2} ; b_{1}, b_{2}, b_{3} ; z\right)$.

Tеорема 3. Для всех $x \in \mathbf{R}$ имеют место тождества

$$
\begin{gathered}
C_{2}(x)=x-\frac{x^{5}}{20} \cdot{ }_{2} F_{3}\left(1, \frac{5}{4} ; \frac{4}{3}, \frac{5}{3}, \frac{9}{4} ;-\frac{x^{4}}{27}\right) ; \\
S_{2}(x)=\frac{x^{3}}{3} \cdot{ }_{2} F_{3}\left(\frac{3}{4}, 1 ; \frac{5}{6}, \frac{7}{6}, \frac{7}{4} ;-\frac{x^{4}}{27}\right) .
\end{gathered}
$$

Доказательство. Для функции $C_{2}(x)$, учитывая (18), получаем соотношения

$$
\begin{gathered}
C_{2}(x)=x-\frac{x^{5}}{2} \sum_{n=0}^{\infty} \frac{(-1)^{n} n !}{(3 n+2) !(4 n+5)} x^{4 n}= \\
=x-\frac{x^{5}}{20}\left(1+\sum_{n=1}^{\infty} \frac{\frac{5}{4} \cdot \frac{9}{4} \cdot \ldots \cdot \frac{4 n+1}{4}}{\left(\frac{4}{3} \cdot \frac{7}{3} \cdot \ldots \cdot \frac{3 n+1}{3}\right)\left(\frac{5}{3} \cdot \frac{8}{3} \cdot \ldots \cdot \frac{3 n+2}{3}\right)\left(\frac{9}{4} \cdot \frac{13}{4} \cdot \ldots \cdot \frac{4 n+1}{4}\right)}\left(-\frac{x^{4}}{27}\right)^{n}\right)= \\
=x-\frac{x^{5}}{20} \sum_{n=0}^{\infty} \frac{1^{\frac{-}{n}}\left(\frac{5}{4}\right)^{\frac{-}{n}}}{\left(\frac{4}{3}\right)^{\bar{n}}\left(\frac{5}{3}\right)^{\bar{n}}\left(\frac{9}{4}\right)^{\bar{n}} n !}\left(-\frac{x^{4}}{27}\right)^{n}=x-\frac{x^{5}}{20} \cdot{ }_{2} F_{3}\left(1, \frac{5}{4} ; \frac{4}{3}, \frac{5}{3}, \frac{9}{4} ;-\frac{x^{4}}{27}\right) .
\end{gathered}
$$

Для функции $S_{1}(x)$, учитывая (19), имеем

$$
S_{2}(x)=x^{3} \sum_{n=0}^{\infty} \frac{(-1)^{n} 16^{n}(2 n) !(3 n) !}{n !(6 n+1) !(4 n+3)} x^{4 n}=
$$




$$
\begin{gathered}
=\frac{x^{3}}{3}+\frac{x^{3}}{3} \sum_{n=1}^{\infty} \frac{\frac{3}{4} \cdot \frac{7}{4} \cdot \ldots \cdot \frac{4 n-1}{4}}{\left(\frac{5}{6} \cdot \frac{11}{6} \cdot \ldots \cdot \frac{6 n-1}{6}\right)\left(\frac{7}{6} \cdot \frac{13}{6} \cdot \ldots \cdot \frac{6 n+1}{6}\right)\left(\frac{7}{4} \cdot \frac{11}{4} \cdot \ldots \cdot \frac{4 n+3}{4}\right)}\left(-\frac{x^{4}}{27}\right)^{n}= \\
=\frac{x^{3}}{3} \sum_{n=0}^{\infty} \frac{\left(\frac{3}{4}\right)^{\bar{n}} 1^{-}}{\left(\frac{5}{6}\right)^{\bar{n}}\left(\frac{7}{6}\right)^{\bar{n}}\left(\frac{7}{4}\right)^{\bar{n}} n !}\left(-\frac{x^{4}}{27}\right)^{n}=\frac{x^{3}}{3} \cdot{ }_{2} F_{3}\left(\frac{3}{4}, 1 ; \frac{5}{6}, \frac{7}{6}, \frac{7}{4} ;-\frac{x^{4}}{27}\right) .
\end{gathered}
$$

На рис. 15 - 20 представлены графики функций комплексного переменного $C_{2}(z), S_{2}(z)$, где $z=x+i y$.

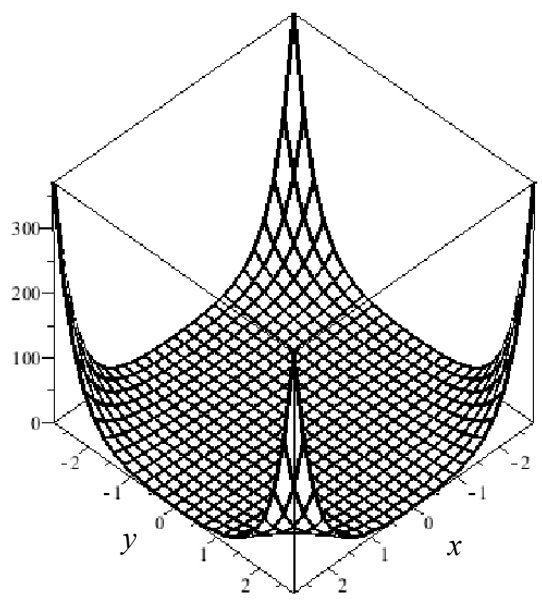

Рис. 15. График функции $\left|C_{2}(z)\right|$

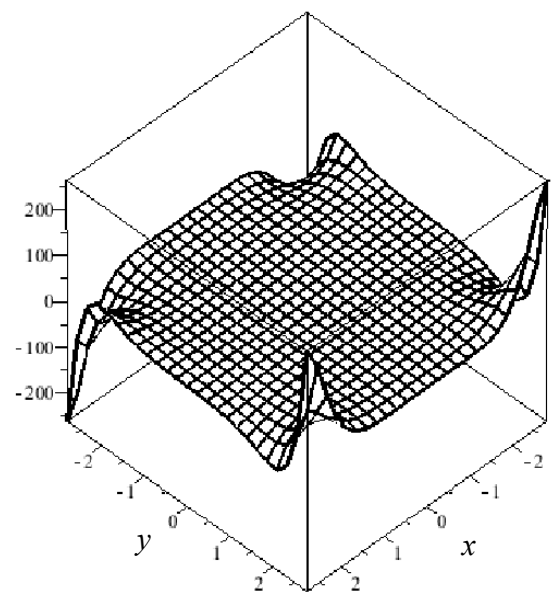

Рис. 17. График функции $\operatorname{Im}\left(C_{2}(z)\right)$

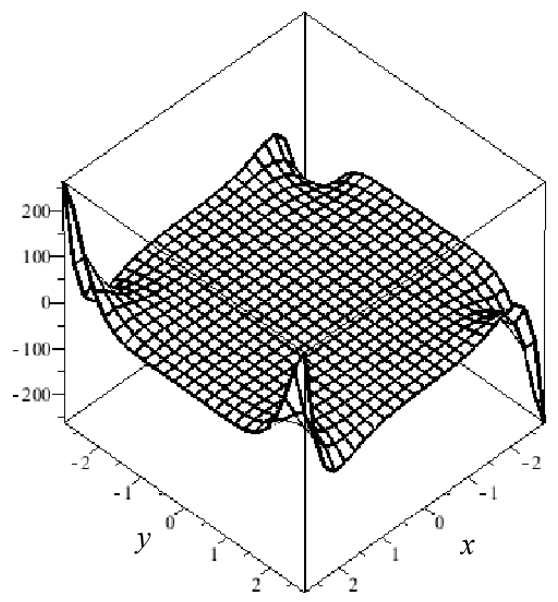

Рис. 16. График функции $\operatorname{Re}\left(C_{2}(z)\right)$

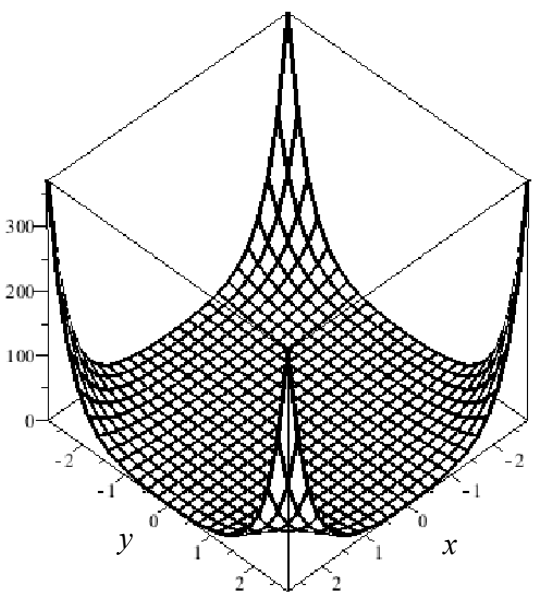

Рис. 18. График функции $\left|S_{2}(z)\right|$ 


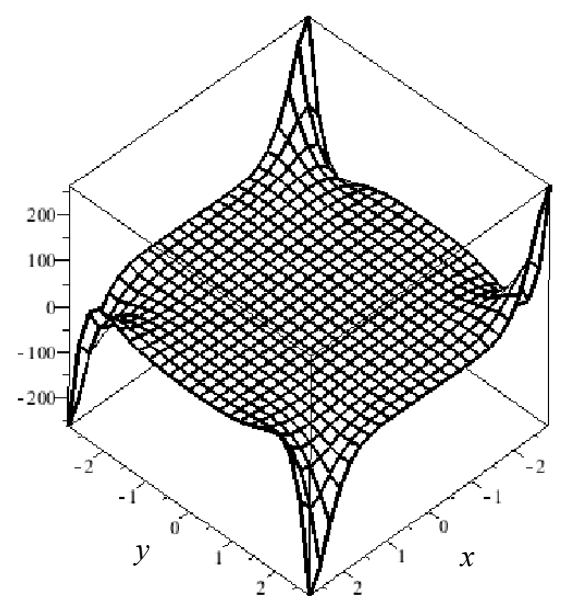

Рис. 19. График функции $\operatorname{Re}\left(S_{2}(z)\right)$

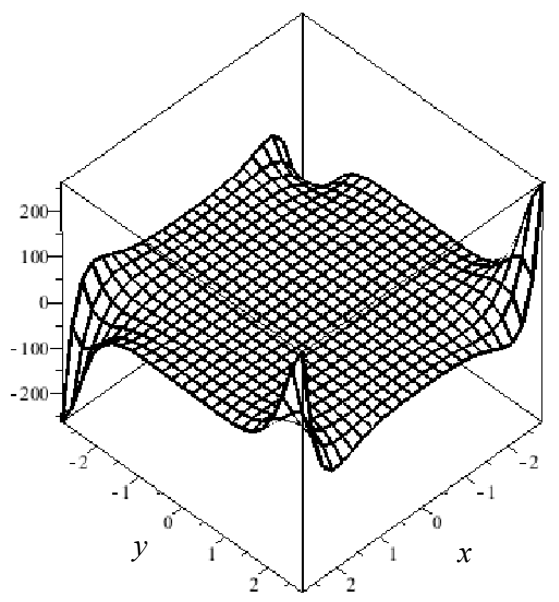

Рис. 20. График функции $\operatorname{Im}\left(S_{2}(z)\right)$

Покажем, что функции $C_{2}(x), S_{2}(x)$ являются решениями задач Коши для обыкновенных линейных дифференциальных уравнений с непрерывными коэффициентами.

Теорема 4. Функиии $C_{2}(x)$ и $S_{2}(x)$ - решения соответственно таких задач Коuи:

$$
\begin{gathered}
27 x^{3} y^{\mathrm{IV}}-135 x^{2} y^{\prime \prime \prime}+\left(16 x^{5}+339\right) y^{\prime \prime}-384 y^{\prime}=-384, \\
y(0)=0, y^{\prime}(0)=1, y^{\prime \prime}(0)=0, y^{\prime \prime \prime}(0)=0 \\
27 x^{3} y^{\mathrm{IV}}-81 x^{2} y^{\prime \prime \prime}+\left(16 x^{5}+177 x\right) y^{\prime \prime}+\left(32 x^{4}-192\right) y^{\prime}=0, \\
y(0)=0, y^{\prime}(0)=0, y^{\prime \prime}(0)=0, y^{\prime \prime \prime}(0)=2 .
\end{gathered}
$$

Доказательство. Из (18) и (19) следует, что функции $C_{2}(x)$ и $S_{2}(x)$ удовлетворяют начальные условия из (22), (23). Покажем, что эти функции являются решениями соответственных дифференциальных уравнений.

Обобщенная гипергеометрическая функция ${ }_{2} F_{3}\left(a_{1}, a_{2} ; b_{1}, b_{2}, b_{3} ; z\right)$, через которую в соответствии с (20) выражается функция $C_{2}(x)$, удовлетворяет обыкновенное линейное дифференциальное уравнение четвертого порядка [16]

$$
\left(\sigma\left(\sigma+b_{1}-1\right)\left(\sigma+b_{2}-1\right)\left(\sigma+b_{3}-1\right)-z\left(\sigma+a_{1}\right)\left(\sigma+a_{2}\right)\right) w(z)=0
$$

где $\sigma$ - дифференциальный оператор $\sigma=z \frac{d}{d z}$. Таким образом, функция

$$
w(z)={ }_{2} F_{3}\left(1, \frac{5}{4} ; \frac{4}{3}, \frac{5}{3}, \frac{9}{4} ; z\right)
$$

из (20) является решением дифференциального уравнения

$$
\left(\sigma\left(\sigma+\frac{1}{3}\right)\left(\sigma+\frac{2}{3}\right)\left(\sigma+\frac{5}{4}\right)-z(\sigma+1)\left(\sigma+\frac{5}{4}\right)\right) w(z)=0
$$


а учитывая, что

$$
\begin{gathered}
\sigma^{2}=z \frac{d}{d z}+z^{2} \frac{d^{2}}{d z^{2}}, \quad \sigma^{3}=z \frac{d}{d z}+3 z^{2} \frac{d^{2}}{d z^{2}}+z^{3} \frac{d^{3}}{d z^{3}}, \\
\sigma^{4}=z \frac{d}{d z}+7 z^{2} \frac{d^{2}}{d z^{2}}+6 z^{3} \frac{d^{3}}{d z^{3}}+z^{4} \frac{d^{4}}{d z^{4}},
\end{gathered}
$$

после несложных преобразований убеждаемся, что она удовлетворяет дифференциальное уравнение

$$
z^{3} w^{\mathrm{IV}}+\frac{33}{4} z^{2} w^{\prime \prime \prime}+\left(\frac{137}{9} z-z^{2}\right) w^{\prime \prime}+\left(5-\frac{13}{4} z\right) w^{\prime}-\frac{5}{4} w=0 .
$$

Произведем в (24) замену независимого переменного по формуле $z=-x^{4} / 27$. Тогда

$$
\begin{gathered}
w_{z}^{\prime}=-\frac{27}{4 x^{3}} w_{x}^{\prime}, \quad w_{z}^{\prime \prime}=\frac{729}{16 x^{7}}\left(x w_{x}^{\prime \prime}-3 w_{x}^{\prime}\right), \quad w_{z}^{\prime \prime \prime}=-\frac{19683}{64 x^{11}}\left(x^{2} w_{x}^{\prime \prime \prime}-9 x w_{x}^{\prime \prime}+21 w_{x}^{\prime}\right), \\
w_{z}^{\mathrm{IV}}=\frac{531441}{256 x^{15}}\left(x^{3} w_{x}^{\mathrm{IV}}-18 x^{2} w_{x}^{\prime \prime \prime}+111 x w_{x}^{\prime \prime}-231 w_{x}^{\prime}\right)
\end{gathered}
$$

и, подставляя в (24), получаем, что функция $w(x)={ }_{2} F_{3}\left(1, \frac{5}{4} ; \frac{4}{3}, \frac{5}{3}, \frac{9}{4} ;-\frac{x^{4}}{27}\right)$ - частное решение линейного однородного уравнения

$$
27 x^{3} w^{\mathrm{IV}}+405 x^{2} w^{\prime \prime \prime}+\left(16 x^{5}+1554\right) w^{\prime \prime}+\left(1386+160 x^{4}\right) w^{\prime}+320 x^{3} w=0 .
$$

Наконец, подставляя в последнее уравнение $w(x)=20 x^{-5}\left(1-C_{2}(x)\right)$, после упрощений получаем, что функция $y=C_{2}(x)$ удовлетворяет дифференциальное уравнение из (22).

Покажем теперь, что функция $S_{2}(x)$ является решением дифференциального уравнения из (23). Аналогично предыдущему доказывается, что обобщенная гипергеометрическая функция

$$
u(z)={ }_{2} F_{3}\left(\frac{3}{4}, 1 ; \frac{5}{6}, \frac{7}{6}, \frac{7}{4} ; z\right)
$$

является решением дифференциального уравнения

$$
z^{3} u^{\mathrm{IV}}+\frac{27}{4} z^{2} u^{\prime \prime \prime}+\left(\frac{83}{9} z-z^{2}\right) u^{\prime \prime}+\left(\frac{245}{144}-\frac{11}{4} z\right) u^{\prime}-\frac{3}{4} u=0,
$$

а функция $u(x)={ }_{2} F_{3}\left(\frac{3}{4}, 1 ; \frac{5}{6}, \frac{7}{6}, \frac{7}{4} ;-\frac{x^{4}}{27}\right)$ - решением дифференциального уравнения

$$
27 x^{3} u^{\mathrm{IV}}-81 x^{2} u^{\prime \prime \prime}+\left(16 x^{5}+177\right) u^{\prime \prime}+\left(32 x^{4}-192\right) u^{\prime}=0 .
$$

После замены в последнем уравнении $w(x)=3 x^{-3} S_{2}(x)$ (с учетом (21)), получаем, что функция $S_{2}(x)$ удовлетворяет уравнения из (23). 


\section{ЛИТЕРАТУРА}

1. Гой Т.П. Нові функції, породжені зростаючими факторіалами, та їх властивості // Буковинский мат. журн. 2013. Т. 1. № 1-2. С. 28-33.

2. Гой Т.П. Неелементарні функції, породжені центральним факторіальними степенями // Вестник Харьковского нац. университета имени В.Н. Каразина. Серия «Математика, прикладная математика и механика». 2014. № 1133. С. 131-139.

3. Гой Т.П. О центральных факториальных степенях и некоторых их применениях // Межвуз. сб. науч. трудов «Математика и математическое образование. Теория и практика». Ярославль: Изд-во ЯГТУ. 2014. Вып. 9. С. 30-35.

4. Goy T.P., Zatorsky R.A. New integral functions generated by rising factorial powers // Kapпатские мат. публикации. 2013. Т. 5. № 2. С. 217-224.

5. Goy T.P., Zatorsky R.A. On a nonelementary function of the Dawson's integral type // Bectник Киевского нац. университета имени Т. Шевченко. Серия «Физико-математические науки». 2014. Вып. 1. С. 15-19.

6. Гой Т.П. Інтеграли від функцій, породжених зростаючими факторіальними степенями // Таврический вестник информатики и математики. 2014. Т. 24. № 1. С. 14-22.

7. Гой Т.П. Нові функції, означені при допомозі факторіальних степенів // Математическое и компьютерное моделирование. Серия «Физико-математические науки». 2014. Вып. 11. С. 18-29.

8. Гой Т.П. О дифференциальных уравнениях функций, определенных с помощью возрастающих и центральных факториалов // Материалы Междунар. конф. «Современные проблемы анализа динамических систем. Приложения в технике и технологиях». Ч. 1. Воронеж: ФГБОУ ВПО «ВГЛТА», 2014. С. 58-61.

9. Jordan C. Calculus of Finite Differences. N.Y.: Chelsea Publishing, 1939.

10. Steffensen J.F. On the definition of the central factorial// J. Inst. Actuaries. 1933. V. 64. No. 2. P. $165-168$.

11. Грэхем Р., Кнут Д., Паташник О. Конкретная математика. Основания информатики. М.: Мир, 1998. 703 с.

12. Заторський Р.А., Малярчук О.Р. Факторіальні степені та трикутні матриці // Карпатские мат. публикации. 2009. Т.1. № 2. С. 217-224.

13. Comtet L. Advanced Combinatorics: The Art of Finite and Infinite Expansions. D. Reider Publishing, 1974.

14. Roman S. The Umbral Calculus. Academic Press, 1984.

15. Справочник по специальным функциям с формулами, графиками и математическими таблицями / под ред. М. Абрамовиц и И. Стиган. М.: Наука, 1979. 832 с.

16. Бейтмен Г., Эрдейи А. Высшие трансцендентные функции. Том 1. Гипергеометрическая функция. Функции Лежандра. М.: Наука. 1973. 294 с.

Статья поступила 12.09.2015 г.

Goy T.P. SPECIAL FUNCTIONS GENERATED BY RISING AND CENTRAL FACTORIAL POWERS

DOI $10.17223 / 19988621 / 40 / 2$

Replacing in the well-known series $\cos x=\sum_{n=0}^{\infty} \frac{(-1)^{n} x^{2 n}}{(2 n) !}, \quad \sin x=\sum_{n=0}^{\infty} \frac{(-1)^{n} x^{2 n+1}}{(2 n+1) !}$ falling factorial powers $\left(m !=m^{\underline{m}}\right)$ by rising and central factorial powers $\left(m^{\bar{m}}\right.$ and $m^{[m]}$ respectively), we obtain real functions $\operatorname{Cos} x=\sum_{n=0}^{\infty} \frac{(-1)^{n} x^{2 n}}{(2 n)^{2 n}}, \quad \operatorname{Sin} x=\sum_{n=0}^{\infty} \frac{(-1)^{n} x^{2 n+1}}{(2 n+1)^{2 n+1}}, \quad \operatorname{Cosc} x=\sum_{n=0}^{\infty} \frac{(-1)^{n} x^{2 n}}{(2 n)^{[2 n]}}$, and Sinc $x=\sum_{n=0}^{\infty} \frac{(-1)^{n} x^{2 n+1}}{(2 n+1)^{[2 n+1]}}$. 
In this paper, we consider the non-elementary Fresnel-type integral functions $C_{1}(x)=\int_{0}^{x} \operatorname{Cos} t^{2} d t, \quad S_{1}(x)=\int_{0}^{x} \operatorname{Sin} t^{2} d t, \quad C_{2}(x)=\int_{0}^{x} \operatorname{Cosc} t^{2} d t, \quad S_{2}(x)=\int_{0}^{x} \operatorname{Sinc} t^{2} d t$. We prove the following formulas:

$$
\begin{gathered}
C_{1}(x)=4\left(\cos \frac{x^{2}}{4} C\left(\frac{x}{2}\right)+\sin \frac{x^{2}}{4} S\left(\frac{x}{2}\right)\right)-x, S_{1}(x)=4\left(\sin \frac{x^{2}}{4} C\left(\frac{x}{2}\right)-\cos \frac{x^{2}}{4} S\left(\frac{x}{2}\right)\right), \\
C_{2}(x)=x-\frac{x^{5}}{20}{ }_{2} F_{3}\left(1, \frac{5}{4} ; \frac{4}{3}, \frac{5}{3}, \frac{9}{4} ;-\frac{x^{4}}{27}\right), S_{2}(x)=\frac{x^{3}}{3}{ }_{2} F_{3}\left(\frac{3}{4}, 1 ; \frac{5}{6}, \frac{7}{6}, \frac{7}{4} ;-\frac{x^{4}}{27}\right),
\end{gathered}
$$

where $C(p)$ and $S(p)$ are Fresnel integrals and ${ }_{2} F_{3}\left(a_{1}, a_{2} ; b_{1}, b_{2}, b_{3} ; z\right)$ is a generalized hypergeometric function.

We also show that functions $C_{1}(x), S_{1}(x)$ are solutions of the ordinary linear second-order differential equations $4 x y^{\prime \prime}-4 y^{\prime}+x^{3} y=-x^{4}-4$ and $4 x y^{\prime \prime}-4 y^{\prime}+x^{3} y=4 x^{2}$, respectively, and the functions $C_{2}(x), S_{2}(x)$ are solutions of the ordinary linear fourth-order differential equations $27 x^{3} y^{\mathrm{IV}}-135 x^{2} y^{\prime \prime \prime}+\left(16 x^{5}+339\right) y^{\prime \prime}-384 y^{\prime}=-384$ and $27 x^{3} y^{\mathrm{IV}}-81 x^{2} y^{\prime \prime \prime}+\left(16 x^{5}+177 x\right) y^{\prime \prime}+$ $+\left(32 x^{4}-192\right) y^{\prime}=0$, respectively.

Keywords: rising factorial power, central factorial power, Fresnel integrals, generalized hypergeometric function, Cauchy problem.

GOY Taras Petrovych (Candidate of Physics and Mathematics, Vasyl Stefanyk Precarpathian National University, Ivano-Frankivsk, Ukraine)

E-mail: tarasgoy@yahoo.com

\section{REFERENCES}

1. Goy T.P., Zatorsky R.A. (2013) New functions generated by rising factorial powers and their properties. Bukovyna Math. Journal. 1(1-2). pp. 28-33. (In Ukrainian)

2. Goy T.P. (2014) Non-elementary functions generated by central factorial powers. Bulletin of V.N. Karazin Kharkiv National University. Series: Mathematics, Applied Mathematics and Mechanics. 1133. pp. 131-139. (In Ukrainian)

3. Goy T.P. (2014) O tsentral'nykh faktorial'nykh stepenyakh i nekotorykh ikh primeneniyakh [On central factorial powers and some of their applications]. In: Matematika i matematicheskoe obrazovanie. Teoriya i praktika [Mathematics and mathematical education. Theory and practice]. Yaroslavl: YSTU. 9. pp. 30-35.

4. Goy T.P., Zatorsky R.A. (2013) New integral functions generated by rising factorial powers. Carpathian Math. Publ. 5(2). pp. 217-224. DOI: 10.15330/cmp.5.2.217-224.

5. Goy T.P., Zatorsky R.A. (2014) On a nonelementary function of the Dawson's integral type. Bulletin of Taras Shevchenko Kyiv National University. Series: Physics and Mathematics. 1. pp. 15-19.

6. Goy T.P. (2014) Integrals of functions generated by rising factorial powers. Taurida Journal of Computer Science and Mathematics. 24(1). pp. 14-22. (in Ukrainian)

7. Goy T.P. (2014) New functions defined by factorial powers. Mathematical and Computer Modelling. Series: Physical and mathematical sciences. 11. pp. 18-29. (in Ukrainian)

8. Goy T.P. (2014) O differentsial'nykh uravneniyakh funktsiy, opredelennykh s pomoshch'yu vozrastayushchikh i tsentral'nykh faktorialov [On differential equations of functions defined by rising and central factorials]. In: Proc. of the International Conference "Sovremenne problemy analiza dinamicheskikh sistem. Prilozheniya v tekhnike i tekhnologiyakh" [Proc. Intern. Conf. "Modern Problems of the Analysis of Dynamic Systems. Appendices in Equipment Technologies"]. Pt 1. Voronezh: VSFA. pp. 58-61. DOI: 10.12737/4702. 
9. Jordan C. (1939) Calculus of Finite Differences. N.Y.: Chelsea Publishing.

10. Steffensen J.F. (1933) On the definition of the central factorial. J. Inst. Actuaries. 64(2). pp. $165-168$.

11. Graham R.L., Knuth D.E., Patashnik O. (1994) Concrete Mathematics. A Foundation for Computer Science. Addison-Wesley.

12. Zatorsky R.A., Malarchuk A.R. (2009) Factorial powers and triangular matrices. Carpathian Math. Publ. 1(2). pp. 161-171. (In Ukrainian)

13. Comtet L. (1974) Advanced Combinatorics: The Art of Finite and Infinite Expansions. D. Reider Publishing.

14. Roman S. (1984) The Umbral Calculus. Academic Press.

15. Abramowitz M., Stegun I.A. (eds.) (1972) Handbook of Mathematical Functions with Formulas, Graphs, and Mathematical Tables. Dover Publication.

16. Bateman H., Erdelyi A. (1953) Higher Transcendental Functions. Vol. 1. McGraw-Hill. 